\title{
METALLIC PAINT APPEARANCE MEASUREMENT AND RENDERING
}

\author{
ROMAN ĎURIKOVIČ AND ANDREJ MIHÁLIK
}

\begin{abstract}
Humans recognize objects visually on the basis of material composition as well as shape. To acquire a certain level of photorealism, it is necessary to analyze, how the materials scatter the incident light. The key quantity for expressing the directional optical effect of materials on the incident radiance is the bidirectional reflectance distribution function (BRDF). Our work is devoted to the BRDF measurements, in order to render the synthetic images, mostly of the metallic paints. We measured the spectral reflectance off multiple paint samples then used the measured data to fit the analytical BRDF model, in order to acquire its parameters. In this paper we describe the methodology of the image synthesis from measured data. Materials such as the metallic paints exhibit a sparkling effect caused by the metallic particles scattered within the paint volume. Our analysis of sparkling effect is based on the processing of the multiple photographs. Results of analysis and the measurements were incorporated into the rendering process of car paint.
\end{abstract}

Mathematics Subject Classification 2000: 65C20, 78M22, 93A30

Additional Key Words and Phrases: BRDF, texture, rendering, metallic paint, measurements

\section{INTRODUCTION}

The appearance of all objects is determined by the how object surface scatters incident light [Tonsho et al. 2001]. To measure the surface properties, a gonio-spectrophotometer device [Achutha 2006] that measures the spectral distribution of reflected radiant power as a function of the illumination and observation angles, is used. The device consists of a light source aperture and a receptor aperture. Mechanical elements have four degrees of freedom to measure the complete reflectance function by moving the receptor aperture and the light source. The disadvantages of the device are their time inefficiency and inaccuracy related to their mechanism.

To decrease the degrees of freedom, the spherical sample rather then a planar one can be used [Kim et al. 2008]. This allows to keep the camera (receptor aperture) in fixed position. Furthermore, the system without any mechanical element was constructed [Rump et al. 2008]. This system consists of multiple cameras mounted on a hemispherical gantry and each camera is equipped with a flash unit. 


\section{R. ĎURIKOVIČ, A. MIHÁLIK}

A large database of tabular BRDF of spherical samples is available online [Matusik et al. 2003]. Unfortunately, it is hard to fit the data in to the rendering pipeline and it is difficult to use it with importance sampling methods [Bagher et al. 2012]. Hence, the analytical representation of BRDF is more common then tabular representation.

There are variety of analytical models, that describe the light reflection off the surface [Ashikhmin and Shirley 2000]. An example is the empirical Wards's model [Ward 1992], that uses the combination of functions capturing the reflection attributes such as the diffuse reflectance in all directions or the concentration of light scattering in a direction near the specular direction for glossy materials. Besides empirical models there are the models, that apply the basic principles of physics to the surface microscopic structure, such as the Cook-Torrance's model [Cook and Torrance 1982].

To make the products visually more appealing, producers, especially in the automotive industry, tend to utilize the glittering effects of metallic paints. Metallic paint coatings, that exhibit sparkling and depth effects, contain metallic flakes [Ďurikovič 2002], [Ďurikovič and Martens 2003]. The flake faces act as tiny mirrors. The ray reflected by a flake can reach observer either directly, or after scattering. Each reflection then substantially attenuates the light energy. Flakes seen directly are the brightest spots, that look like sparkles [Sergey Ershov 1999].

Metallic paints have different micro and macro appearance. Macro appearance, when the paint is being observed from far distance, can be described by the BRDF, on the other hand the micro appearance can be represented by the irregular random texture that looks like bright sparkles dusted on the paint. Commonly, the statistical characteristics such as fluctuations due to scattering by flakes are estimated and then the paint texture is reproduced by superimposing random fluctuations on the image [Sergey Ershov 1999]. That method, however, does not focus on rendering of sparkles itself and the approach is limited to static scenes with pre-computed procedural texture.

Another approach [Ďurikovič and Martens 2003] uses the explicit modeling of sparkles by the given geometrical shape and distribution along the model surface. This will allow to render the dynamic scenes with camera zoom in, zoom out, and stereo observation of the scene with sparkling effect. The draw back is that the proposed method is not a real time rendering approach but it can be implemented easily.

We propose the design and methodology devoted to the measurement of spectral reflectance from material samples in order to render photo-realistic images. We propose a simple device, that allows us to measure spectral reflectance under arbitrary angle in the plane of incidence. We fit the unknown parameters of the analytical BRDF model to the measured spectral data. We performed fitting by the nonlinear least square optimization [Bjorck 1996]. To validate the fitting results we utilized our virtual gonio-spectrophotometer. In order to render sparkles, we captured the texture of a car paint sample.

The remaining of this paper is organized as follows. First we define the basic terminology of reflectance function, Section 1.1. Following Section 2 describes how to convert the measured reflectance into a BRDF. In Section 3 we introduce the set up of our measurement device. In the Section 4 we introduce the process of BRDF measurement. Section 5 describes an acquisition of analytical BRDF model from the measured data. In the Section 


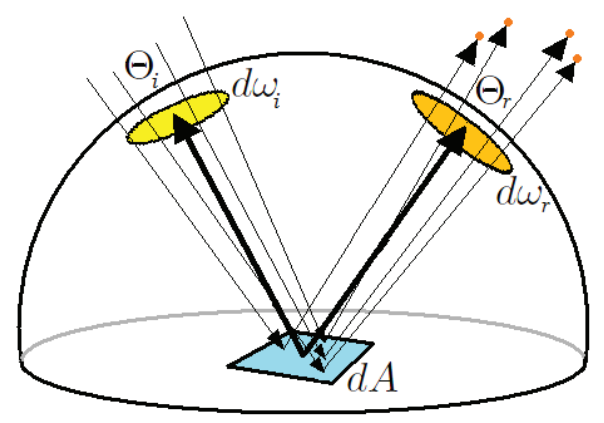

Fig. 1. Bidirectional reflectance function. Flux of the light entered unit sphere through $d \omega_{i}$ and reflected from the area $d A$ through solid angle $d \omega_{r}$.

6 we describe modeling of sparkling and glittering effects. Section 7 introduces the rendering process utilizing graphics processing unit (GPU) and in Section 8 we conclude our work.

\subsection{Reflectance Function}

A mathematical representation of surface reflection behavior can be achieved by considering a number of variables such as wavelength, angle of incidence, observation position etc. A common approach is to use a simplified model by considering the directional and spectral properties of reflection. Bidirectional reflectance distribution function (BRDF) is defined by the ratio of outgoing radiance and incident irradiance [Rushmeier 2001], i.e.,

$$
f_{r}\left(\lambda, \mathbf{x}, \Theta_{i} \rightarrow \Theta_{r}\right)=\frac{L_{r}}{E_{i}}=\frac{L_{r}\left(\lambda, \mathbf{x} \rightarrow \Theta_{r}\right)}{L_{i}\left(\lambda, \mathbf{x} \leftarrow \Theta_{i}\right) \cos \theta_{i} d \omega_{i}},
$$

where $\lambda$ is wavelength of the light. Index $i$ indicates incident light, whereas the index $r$ indicates reflected light (see Figure 1). $\Theta=(\theta, \varphi)$ denotes the light direction, whereas $\theta$ and $\varphi$ are spherical coordinates and $\theta$ is angle between the surface normal and particular light direction. Arrow notation indicates the direction of light transport i.e. $\mathbf{x} \leftarrow \Theta_{i}$ says the light traveling from the incident direction $\Theta_{i}$ hits the surface point $\mathbf{x}$.

Solid angle is defined by the projected area of a surface patch onto a unit sphere of a point, meaning that a solid angle is subtended by a point, $S_{0}$, and a surface patch, $d A$ :

$$
d \omega=\frac{d A \cos \theta}{r^{2}}
$$

where $d A \cos \theta$ is the foreshortened area of $d A$ on the direction of $S_{0}$. The distance between $S_{0}$ and $d A$ is $r$, and the angle between surface normal $\mathbf{n}$ and the point direction is $\theta$.

Radiance. Frequently, we use outgoing radiance to measure the lighting sources, but use irradiance to measure the lighting intensity received by a patch. By definition, the radiance of an area lighting source is

$$
L_{r}(\lambda, \mathbf{x} \rightarrow \Theta)=\frac{d \Phi_{r}\left(\lambda, \mathbf{x} \rightarrow \Theta_{r}\right)}{\cos \theta_{r} d A_{r} d \omega_{r} d \lambda},
$$



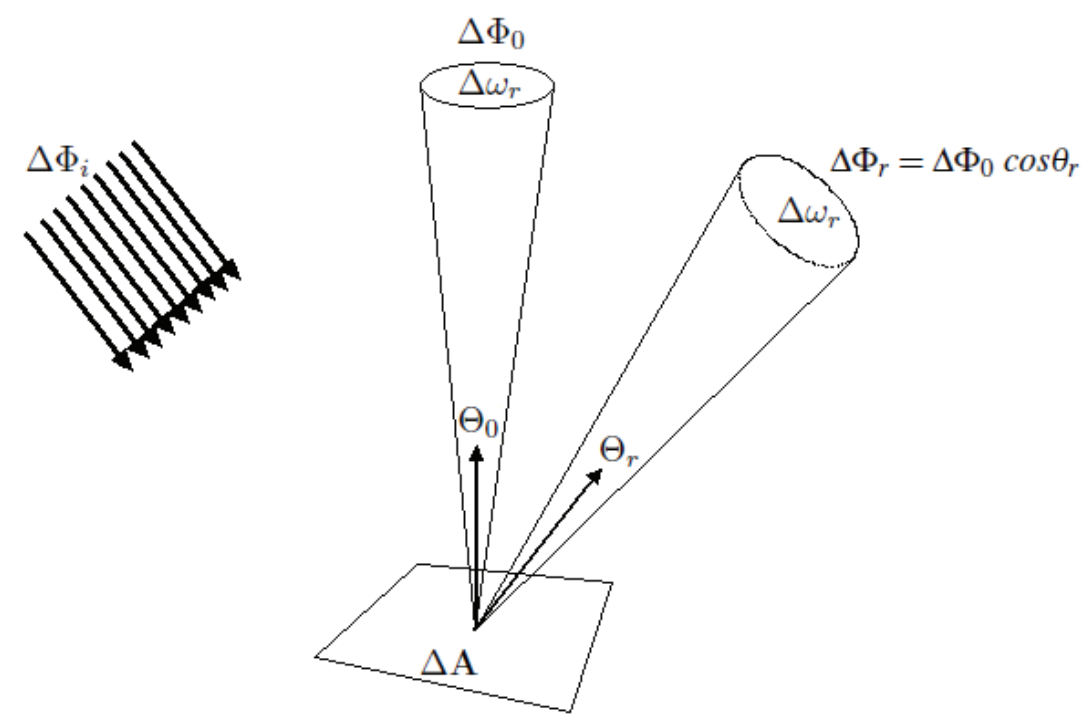

Fig. 2. Reflected parallel light from the surface to the receptor aperture through the solid angle. $\Theta_{0}$ is the direction of surface normal, whereas $\Theta_{r}$ is arbitrary direction.

where $\Phi_{r}$ is the radiant power (flux), $d A_{r}=d A$ is the fragment of lighting surface area around the location point $\mathbf{x}, \Theta_{r}=\left(\theta_{r}, \varphi_{r}\right)$ is used to represent the lighting direction, and $d \omega_{r}$ is solid angle fragment.

Irradiance. On the other hand, we use irradiance to represent the lighting received by a surface patch $d A_{i}$. By definition,

$$
E_{i}=\frac{d \Phi_{i}\left(\lambda, \mathbf{x} \leftarrow \Theta_{i}\right)}{d A_{i}} .
$$

\section{CONVERSION OF REFLECTANCE TO BRDF}

Reflectance is basically the ratio of energy reflected off the surface to the incident energy. We are able to measure the reflectance using measuring devices. However, a BRDF differs from the reflectance because; the BRDF is not really a function itself, but a distribution or generalized function. Like a probability density, a BRDF can contain distributions that make sense only inside integrals, but correspond to straightforward computational operations. In this section we propose a methodology to covert the reflectance measurements from a device to a values of BRDF.

PROPOSITION 1. To acquire the BRDF of diffuse surface we can measure reflectance using directional light source, see Figure 2. Then the estimation of BRDF could be done by the following formula:

$$
f_{r}\left(\mathbf{x}, \Theta_{i} \rightarrow \Theta_{r}\right) \approx \frac{1}{\cos \theta_{r} \Delta \omega_{r}} \frac{\Delta \Phi_{r}}{\Delta \Phi_{i}},
$$




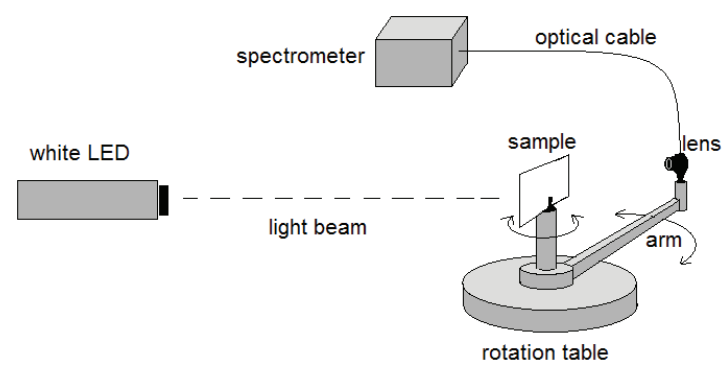

Fig. 3. Device setup for the reflectance measurements of material samples.

where $\Delta \omega_{r}$ is the projected area of receptor aperture to the unit sphere. Angle between surface normal and direction to the receptor is $\theta_{r}$. Total flux reflected from the spot at the surface of area $\Delta \mathbf{A}$ is denoted as $\Delta \Phi_{r}$, whereas total flux of incident light at that spot is denoted as $\Delta \Phi_{i}$.

PROOF. We can prove above statement for the diffuse surfaces by the following consideration. Substitute the definitions from Eqn. 3, 4 into a BRDF definition Eqn. 1:

$$
f_{r}\left(\mathbf{x}, \Theta_{i} \rightarrow \Theta_{r}\right)=\frac{L_{r}}{E_{i}}=\frac{\frac{d \Phi_{r}\left(\mathbf{x} \rightarrow \Theta_{r}\right)}{\cos \theta_{r} d A_{r} d \omega_{r}}}{\frac{d \Phi_{i}\left(\mathbf{x} \leftarrow \Theta_{i}\right)}{d A_{i}}}=\frac{d \Phi_{r}}{d \Phi_{i}} \frac{d A_{i}}{\cos \theta_{r} d \omega_{r} d A_{r}} .
$$

Lighting is received by a surface patch $d A_{i}=\Delta A$ on the other hand the surface patch $d A_{r}=$ $\Delta A$ is illuminating the receptor in solid angle $d \omega_{r}$. Assume that solid angle subtended by the receptor is $d \omega_{r}=\Delta \omega_{r}$ the last term simplifies to

$$
f_{r}\left(\mathbf{x}, \Theta_{i} \rightarrow \Theta_{r}\right)=\frac{1}{\cos \theta_{r} \Delta \omega_{r}} \frac{\Delta \Phi_{r}}{\Delta \Phi_{i}} .
$$

\section{DEVICE SETUP}

Spectral reflectance measurement device with two degrees of freedom, Fig. 3, consists of the fixed light source and receptor moving around the sample. Light source is the LED STAR 2,5 WHITE $120 \mathrm{LM} / 120^{\circ}$ LAMBERTIAN with power input $2.5 \mathrm{~W}$, luminous flux $100-120 \mathrm{~lm}$, radiation angle $120^{\circ}$ and color temperature $6500-8000 \mathrm{~K}$. The receptor is connected by the optical cable to the spectrometer Solar S100 (grating 3001/mm, TCD linear image sensor, spectral range 190-1100nm, spectral resolution 1nm, dynamic range 900:1). The distance between the source and the sample is $90 \mathrm{~cm}$. The sample itself can be rotated in arbitrary angles. Receptor with mounted lens is located on the arm within the $40 \mathrm{~cm}$ distance from the sample.

The device calibration is done before the actual BRDF measurements. First, we capture the background noise with spectrometer. Second, we subtract the background noise from all measurements. Similarly since, the exposure curve of spectrometer is linear, we also divide the resulting current value by the exposure time. The light source spectral power density, $\Delta \Phi_{i}$ in Eqn. 5, is captured by the spectrometer using a perfect mirror. 


\section{R. ĎURIKOVIČ, A. MIHÁLIK}

\section{BRDF MEASUREMENTS}

In this section we describe how to measure the BRDF of diffuse and specular surfaces. The diffuse BRDF is estimated from the ratio of reflected flux $\Delta \Phi_{r}$ and the light source spectral power density $\Delta \Phi_{i}$. The BRDF of specular surface is estimated from the ratio of reflected flux from sample, $d \Phi_{r}$, and the flux reflected from the white diffuse standart, $\Delta \Phi_{r_{s}}$.

\subsection{Diffuse surfaces}

In order to measure the diffuse surface we set the exposure time to $2999 \mathrm{~ms}$. Diffuse sample surface is illuminated under the angle of incidence $20^{\circ}$ and the receptor aperture measures the reflected flux, $\Delta \Phi_{r}$, in normal direction, i.e. $0^{\circ}$. Finally, the BRDF is evaluated for every given wavelength by substituting light source spectral power density, $\Delta \Phi_{i}$, to Eqn. 5 .

The solid angle from Eqn. 5 can be approximated as

$$
\Delta \omega_{r} \approx \frac{\pi w^{2}}{4 d^{2}}
$$

where $d=40 \mathrm{~cm}$ is distance from the sample to the receptor aperture and $w=0.4 \mathrm{~cm}$ is the diameter of aperture. We measured the spectral distribution from the diffuse surface in the band from $380 \mathrm{~nm}$ to $780 \mathrm{~nm}$ with the interval step $5 \mathrm{~nm}$.

\subsection{Metallic surfaces}

The BRDF measurement of metallic surface is more complicated. However, if we know the BRDF values of a sample surface, we can derive the BRDF values of metallic sample using the measured reflectance of both samples. We choose the reference sample a white diffuse surface standard and measure the sample BRDF, $f_{r_{s}}$. Finally, we derive the BRDF, $f_{r}$, of the metallic sample using the following ratio [Ward 1992]:

$$
f_{r}\left(\mathbf{x}, \Theta_{i} \rightarrow \Theta_{r}\right)=\frac{d \frac{d d \Phi_{r}}{d A \cos \theta_{r} d \omega_{r}}}{d E_{i}\left(\mathbf{x} \leftarrow \Theta_{i}\right)}=\frac{d \Phi_{r}}{d \Phi_{r_{s}}} \frac{d \frac{d d \Phi_{r_{s}}}{d A \cos \theta_{r} d \omega_{r}}}{d E_{i}\left(\mathbf{x} \leftarrow \Theta_{i}\right)}=\frac{d \Phi_{r}}{d \Phi_{r_{s}}} f_{r_{s}}\left(\mathbf{x}, \Theta_{i} \rightarrow \Theta_{r}\right),
$$

where the BRDF of the standard is $f_{r_{s}}, \Phi_{r_{s}}$ is reflected flux off the standard and $\Phi_{r}$ is flux reflected of measured metallic sample under the same directions.

We have measured two car paint samples, copper surface and the white diffuse standard. The reflectance was measured under the angles of incidence $\theta_{i} \in\left\{20^{\circ}, 60^{\circ}\right\}$. The receptor was positioned to obtain reflected flux off the surface under the angles $\theta_{r} \in$ $\left\{10^{\circ}, 20^{\circ}, 30^{\circ}, 40^{\circ}, 50^{\circ}, 60^{\circ}, 70^{\circ}\right\}$ to the surface normal in the plane of incidence. The exposure time for the mirror reflection is set to $102 \mathrm{~ms}$ to decrees the highlight and for off-specular reflection to $2999 \mathrm{~ms}$. Total reflected flux is calculated for all wavelengths in the visible band $380 \mathrm{~nm}$ to $780 \mathrm{~nm}$ sampled by $5 \mathrm{~nm}$ using Eqn. 8 .

\section{FITTING MODEL PARAMETERS}

To achieve realistic appearance of material surfaces in real-time rendering the analytical models are commonly used. In particular the Cook-Torrance model is a physically-based 
microfacet model that is focused on (glossy) specular reflection. It uses the surface roughness model developed by Torrance \& Sparrow in [Torrance and Sparrow 1967]. This model treats surface as a collection of microscopic facets. The macroscopic optical properties of a surface are then analytically derived from properties of individual facets and statistical distributions of such properties.

\subsection{Analytical BRDF Model}

Although, the surface has a normal $\mathbf{N}$, at a microscopic level the surface has height variations that result in many different surface orientations at a detailed level. At the perfectly flat surface a viewer is able to see light source reflection, if angular bisector $\mathbf{H}$ of light and viewing direction is in the direction of surface normal $\mathbf{N}$. However, due to different surface orientations at a detailed level, light source may be partially seen at surface positions where $\mathbf{H}$ is not at direction of the surface normal. At this particular positions, however, $\mathbf{H}$ has the same direction as a microfacet normal.

A statistical model of the variation in surface height generally takes the form of giving the distribution of facets that have a particular slope. Most commonly used is the Beckmann distribution function based on physical theory on scattering of electromagnetic waves:

$$
D=\frac{e^{-\frac{\tan ^{2} \alpha}{m^{2}}}}{m^{2} \cos ^{4} \alpha},
$$

where $\alpha$ is the angle between surface and facet normal and $m$ is the root mean square slope of microfacets parameterizing the surface's roughness.

If we assume V-grooved surface, then we need to take into account with self shadowing and masking. The geometric attenuation factor $G$ models the geometric effects shadowing and masking between microfacets that occur at larger angles of incidence or reflection. It is defined by the formula:

$$
G=\min \left(1, \frac{2(\mathbf{H} \cdot \mathbf{N})(\mathbf{V} \cdot \mathbf{N})}{\mathbf{H} \cdot \mathbf{V}}, \frac{2(\mathbf{H} \cdot \mathbf{N})(\mathbf{L} \cdot \mathbf{N})}{\mathbf{H} \cdot \mathbf{V}}\right),
$$

where $\mathbf{L}$ and $\mathbf{V}$ are the unit vectors in the direction to the light source $\Theta_{i}$ and in the direction of observation $\Theta_{r}$, respectively.

The Cook-Torrance model provides a good reproduction of the appearance of many real materials. Especially metallic surfaces profit from the increased realism of the specular factor. Effects like the characteristic color shift towards the color of the incident light near grazing angles and the off-specular peak for very rough surfaces greatly improve the perceived realism of renderings. The off-specular peaks are the consequence of shadowing and masking causing asymmetries.

BRDF of the Cook-Torrance can be compactly written as:

$$
f_{r}\left(\mathbf{x}, \Theta_{i} \rightarrow \Theta_{r}\right)=\frac{k_{d}}{\pi}+k_{s} \frac{F_{r}\left(\theta_{h}\right) D G}{\pi\left(\cos \theta_{i}\right)\left(\cos \theta_{r}\right)},
$$

where $\theta_{h}$ is angle between $\mathbf{L}$ and $\mathbf{H}, \theta_{i}$ is angle between $\mathbf{L}$ and $\mathbf{N}$ and $\theta_{r}$ is angle between $\mathbf{V}$ and $\mathbf{N}$. $F_{r}$ is the reflection coefficient which gives the fraction of incident light that 


\section{R. ĎURIKOVIČ, A. MIHÁLIK}

is reflected from the surface and stems from the Fresnel equations. To model Fresnel reflectance we use Schlick approximation:

$$
F_{r}(\theta)=F_{0}+\left(1-F_{0}\right)(1-\cos \theta)^{5},
$$

where

$$
F_{0}=\left(\frac{1-\frac{n_{1}}{n_{2}}}{1+\frac{n_{1}}{n_{2}}}\right)^{2}
$$

is reflectance of incident light from the direction parallel to the surface normal. $n_{1}$ nad $n_{2}$ are indices of refraction of the volume above the surface and the material beneath, respectively.

\subsection{Analytical BRDF Model}

To obtain analytical model based on measured data we have to perform fitting algorithm. Fitting analytical reflectance models is usually done in two steps [Ngan et al. 2005]. First compute diffuse and specular RGB coefficients using a linear least square optimization, then compute the model parameters through second optimization.

In our approach we computed the reflectance model parameters separately for each wavelength. This allows us to incorporate wavelength effects. As the fitting algorithm we used Levenberg-Marquardt optimization algorithm implemented in the open source numerical analysis and data processing library ALGLIB [Bochkanov and Bystritsky 2013].

The least squares curve fitting problem is to find the parameters $\mathbf{p}$ minimizing the sum of squares errors:

$$
\min _{\mathbf{p}} \sum_{j=1}^{n}\left(y_{j}-f\left(\mathbf{x}_{j}, \mathbf{p}\right)\right)^{2}, .
$$

where $y_{j}$ are the estimated BRDF values. We approximate the measured BRDF by the Cook-Torrance's analytical model (see Equation 11). In order to acquire its parameters $\mathbf{p}=\left(k_{d}, k_{s}, m, F_{0}\right)$ for the particular wavelength we utilized Cook-Torrance's function $f_{r}$ as the function representing analytical model with unknown parameters. After total number of measurements $n(2 \times 7$, we have 2 angles of incidence and 7 receptor positions), we assigned resulting measured BRDF to the dependent variable $y_{j}$. Independent variables represent angles of measurements $\mathbf{x}_{j}=\left(\theta_{i_{j}}, \theta_{r_{j}}\right)$. We performed the fitting for each wavelength in the band from $380 \mathrm{~nm}$ to $750 \mathrm{~nm}$ with interval step $5 \mathrm{~nm}$ separately. Hence, as a result of optimization, we got wavelength dependent parameters $k_{d}(\lambda), k_{s}(\lambda), m(\lambda)$ and $F_{0}(\lambda)$ of Cook-Torrance BRDF for particular wavelength.

\subsection{Validation}

We compared the measured reflectance with the reflectance computed by the virtual goniospectrophotometer [Mihálik and Ďurikovič 2011]. Compared measurements were taken under the angles $\theta_{i}=\theta_{r}=60^{\circ}$ and $\theta_{i}=\theta_{r}=20^{\circ}$ in the plane of incidence. These angles are prescribed in standardized gloss measurements [ISO2813 1994]. We have measured white diffuse surface sample, copper surface, gray metallic car paint Cendré and blue metallic 
car paint Neysha. Results are in Table I evaluated in the form of the relative root mean square defined by:

$$
\text { relative } \mathrm{RMSE}=\sqrt{\sum_{\lambda}^{n} \frac{100^{2}}{\hat{\Phi}(\lambda)^{2}}(\hat{\Phi}(\lambda)-\Phi(\lambda))^{2}} \%,
$$

where $\hat{\Phi}(\lambda)$ is the reflected flux measured on real sample and $\Phi(\lambda)$ is the flux computed by the virtual gonio-spectrophotometer using acquired reflectance model at the particular wavelength. The sum is computed over the band from $380 \mathrm{~nm}$ to $750 \mathrm{~nm}$ with interval step $5 \mathrm{~nm}$.

\section{SPARKLE MEASUREMENTS}

As mentioned earlier there are basically two approaches to simulate flakes in the paint: procedural texture based on the flakes distribution and explicitly modeled geometry of the flakes. Second approach is more strait forward, but ineffective if we consider real-time animation. Approaches more suitable for real-time rendering involve the sparkle textures created using the distribution of flakes within the paint.

To capture the sparkling effect we take multiple photographs of reflected light from metallic samples. Since, the reflectance of metallic flakes is specular, sparkling effect is highly dependent on the angle of observation and the direction of incident light. Our measurement takes into account the light source position changed in the plane perpendicular to the sample surface and camera positioned in the same plane. Camera view direction is in the direction of surface normal (see Figure 4). Angles of incidence were $\theta_{i} \in\left\{10^{\circ}, 20^{\circ}, 30^{\circ}\right.$, $40^{\circ}, 50^{\circ}, 60^{\circ}, 70^{\circ}$.

Specular surface reflects most intensity when the surface normal is in the same direction as vector $\mathbf{H}$. Vector $\mathbf{H}$ is the halfway vector between the vectors $\mathbf{L}$ and $\mathbf{V}$. All vectors are in the same plane and the angle between $\mathbf{L}$ and $\mathbf{H}$ is same as the angle between $\mathbf{V}$ and $\mathbf{H}$. This motivated us to define the angle:

$$
\theta_{h}=\frac{\theta_{i}}{2} .
$$

Consider facet that reflects the light under the angle of incidence $\theta_{i}$ specularly in the direction of $\mathbf{N}$. Hence the angle between surface normal of this facet and average normal of sample surface $\mathbf{N}$ is $\theta_{h}$.

Every sample image illuminated under the angles of incidence $\theta_{i}$, was cropped to the rectangular area $233 \times 233$. We have applied segmentation techniques to find the pixels with

\begin{tabular}{|l|l|l|}
\hline \multirow{2}{*}{ surface sample } & \multicolumn{2}{|c|}{ relative RMSE (\%) } \\
\cline { 2 - 3 } & $20^{\circ} \rightarrow 20^{\circ}$ & $60^{\circ} \rightarrow 60^{\circ}$ \\
\hline diffuse surface & 2.826 & 1.427 \\
\hline copper & 14.687 & 0.192 \\
\hline Neysha & 4.350 & 5.132 \\
\hline Cendré & 5.695 & 0.335 \\
\hline
\end{tabular}

Table I. Relative root mean square error of the reflectance along the wavelength. 


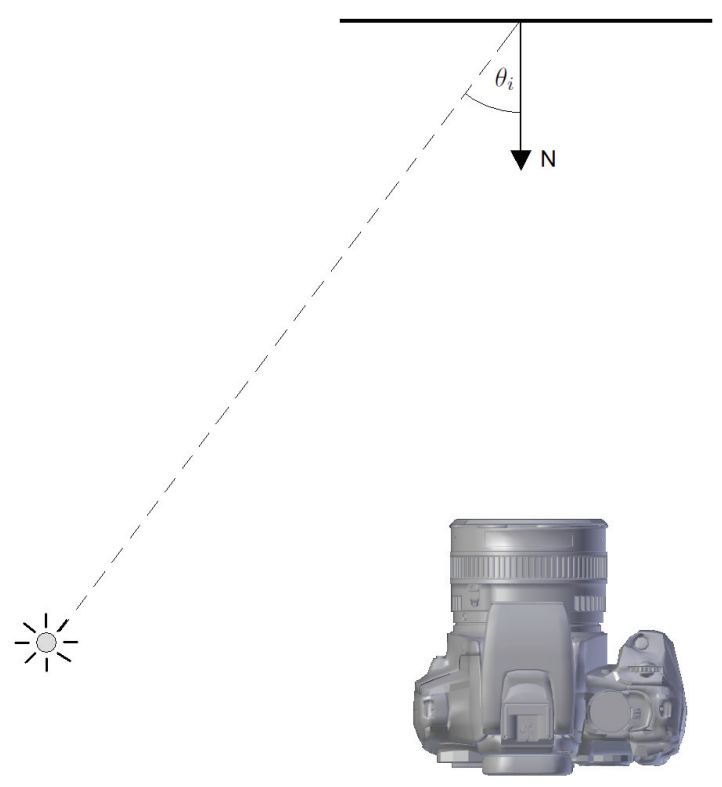

Fig. 4. Photographing of metallic car paint under the multiple angles of incidence.

highest intensities and store them in a bit map, the most intense pixels have assigned value 1. Next we pack all bitmats into a single image texture with 8 bits per pixel.

If we denote $b_{k}(x, y)$ as $k$-th bitmap where $x$ and $y$ are image coordinates and $k \in[0 . .6]$, then for the texture $t(x, y)$ we have

$$
t(x, y)=\sum_{k=0}^{6} b_{k}(x, y) 2^{k} .
$$

Example of 8-bit texture is depicted in Figure 5. Hence, $k-t h$ bit determines if there is a visible sparkle in the $k-t h$ digital image where the angle of incidence was $\theta_{i}=$ $=70^{\circ}-k 10^{\circ}$. Slope from the facet normal to the average surface normal $\mathbf{N}$ is approximated as $\theta_{h}=35^{\circ}-k 5^{\circ}$, according to Eqn. 15. If the facet surface acts as a mirror, the sparkle will be visible when $\cos \theta_{h}=\mathbf{N} \cdot \mathbf{H}$.

\subsection{Sparkle Rendering}

How do we use the above sparkle texture to check if the sparkle is shinning? First, we calculate angle between $\mathbf{N}$ and $\mathbf{H}$ by $\alpha=\arccos (\mathbf{N} \cdot \mathbf{H})$. Knowing the angle $\alpha$, we can find out if there is a visible sparkle at the particular position. We check the texture for particular bit of 8-bit pixel corresponding to angle $\theta_{h}=\alpha$ if it is set to 1 at given coordinates $(x, y)$. The problem is, that during the rendering process, $\alpha$ does not have to reach same value as $\theta_{h}$. We have to include also angles of incidence in between measured ones and attenuate 


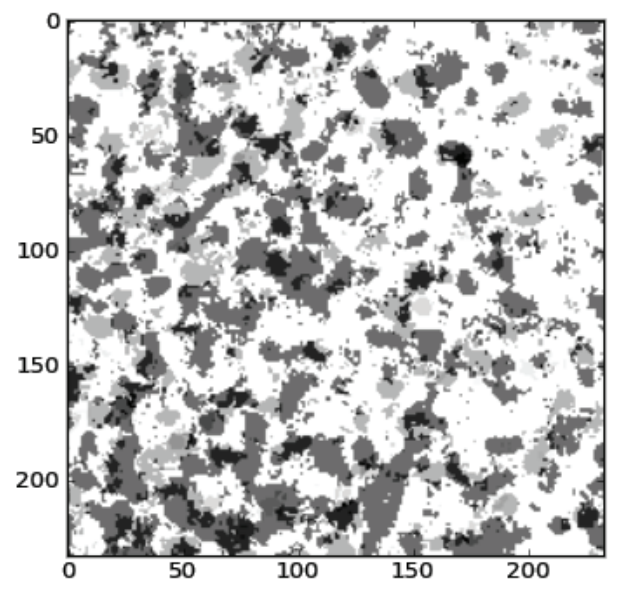

Fig. 5. 8-bit sparkle texture. The level of gray determines $\theta_{h}$ of the sparkle. Smaller values of angle $\theta_{h}$, the image pixel is darker. White color means that there is no sparkle.

the sparkle according the slight change in angles. This idea leads to the following equation

$$
I_{r}=C_{s}\left(\cos \left(\alpha-\theta_{h}\right)\right)^{e},
$$

where $C_{S}$ is the color of sparkles estimated from the digital images. We set $e=24$ that determine the shininess of sparkle. Difference $\alpha-\theta_{h}$ is the approximation of angle between the facet normal and $\mathbf{H}$.

The problem with this method is that the small sparkle texture is repeatedly used to over the object surface and a repeated sparkle pattern can we visible on the surface.

\section{IMPLEMENTATION AND RESULTS}

We measured the spectral reflectance of samples, however common displays support RGB color format. Therefore, it is required to convert spectral reflectance to RGB color space. Our rendering process consisted of three parts. First, the intensity for each wavelength was computed. Since we found the parameters of Cook-Torrance's model, we could compute intensities for each wavelength using this model. Second, we transformed the spectral distribution to CIE XYZ color space and then to RGB color space. Finally, we added the sparkle intensity $I_{r}$ to the resulting color according to the sparkle texture.

\subsection{Implementation of GPU}

Since we are dealt with GPU, we used the 1D floating point texture as a storage of spectral distributions. For each wavelength we stored the particular parameters to the texture as an array. Particularly parameters $k_{d}(\lambda), k_{s}(\lambda), m(\lambda)$ and $F_{0}(\lambda)$ of Cook-Torrance's model that were acquired by the fitting process. For the purpose of the conversion to XYZ color space we had to store also CIE color-matching functions $\bar{x}(\lambda), \bar{y}(\lambda)$ and $\bar{z}(\lambda)$ (see Figure 6). If we denote the texture as $t(s, c)$, where $s \in[0,1]$ and $c \in\{R, G, B\}$ is the channel, then we 


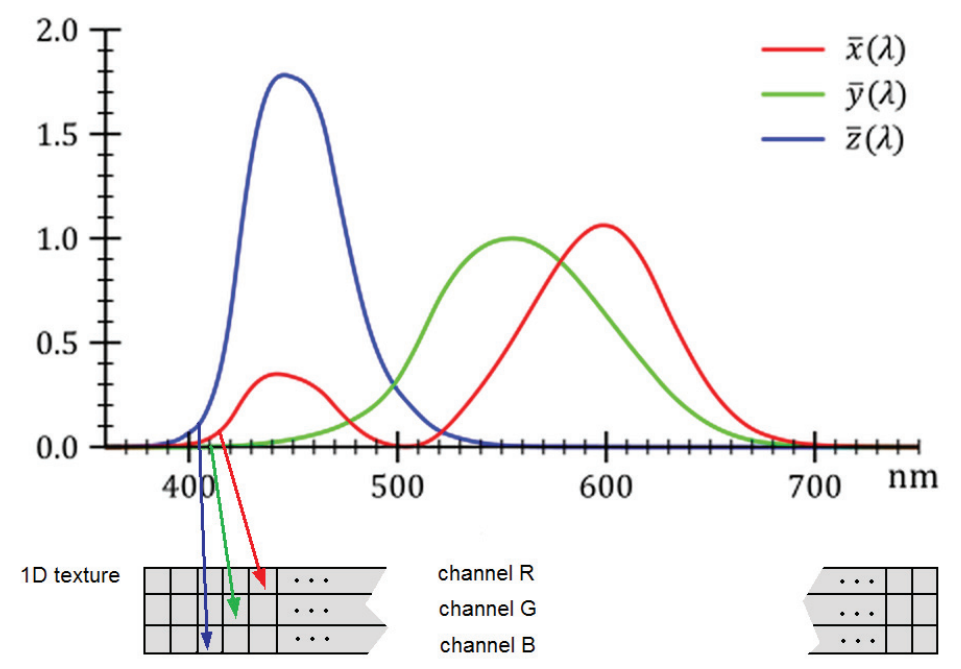

Fig. 6. Mapping the spectral power distribution to the texture as an array.

can map the CIE color-matching functions to the 1D texture as follows:

$$
t\left(\frac{\lambda-380}{780-380}, R\right)=\bar{x}(\lambda), \quad t\left(\frac{\lambda-380}{780-380}, G\right)=\bar{y}(\lambda), \quad t\left(\frac{\lambda-380}{780-380}, B\right)=\bar{z}(\lambda) .
$$

To compute X, Y and Z values of CIE XYZ color model we performed following summation using GPU:

$$
\begin{aligned}
& X=\sum_{\lambda=380}^{780} t\left(\frac{\lambda-380}{780-380}, R\right) L_{r}\left(\lambda, \mathbf{x} \rightarrow \Theta_{r}\right), \\
& Y=\sum_{\lambda=380}^{780} t\left(\frac{\lambda-380}{780-380}, G\right) L_{r}\left(\lambda, \mathbf{x} \rightarrow \Theta_{r}\right), \\
& Z=\sum_{\lambda=380}^{780} t\left(\frac{\lambda-380}{780-380}, B\right) L_{r}\left(\lambda, \mathbf{x} \rightarrow \Theta_{r}\right),
\end{aligned}
$$

where $L_{r}$ is computed radiance given by standart rendering equation using the BRDF, $f_{r}$ from Eqn. 11 with the estimated and stored parameters of Cook-Torrance model for particular wavelength. To obtain RGB values, vector $(\mathrm{X}, \mathrm{Y}, \mathrm{Z})$ is needed to multiply by the $X Y Z$ to $R G B$ transformation matrix:

$$
\left[\begin{array}{l}
R \\
G \\
B
\end{array}\right]=\left[\begin{array}{ccc}
2.3706 & -0.9000 & -0.4706 \\
-0.5138 & 1.4253 & 0.0885 \\
0.0052 & -0.0146 & 1.009
\end{array}\right]\left[\begin{array}{l}
X \\
Y \\
Z
\end{array}\right]
$$

We measured and then rendered two metallic car paints sample. The sample called Neysha had blue basecoat with small metallic grains. The sample called Cendré contained gray pigments. The rendered results of these metallic pains are shown in Figure 7. 

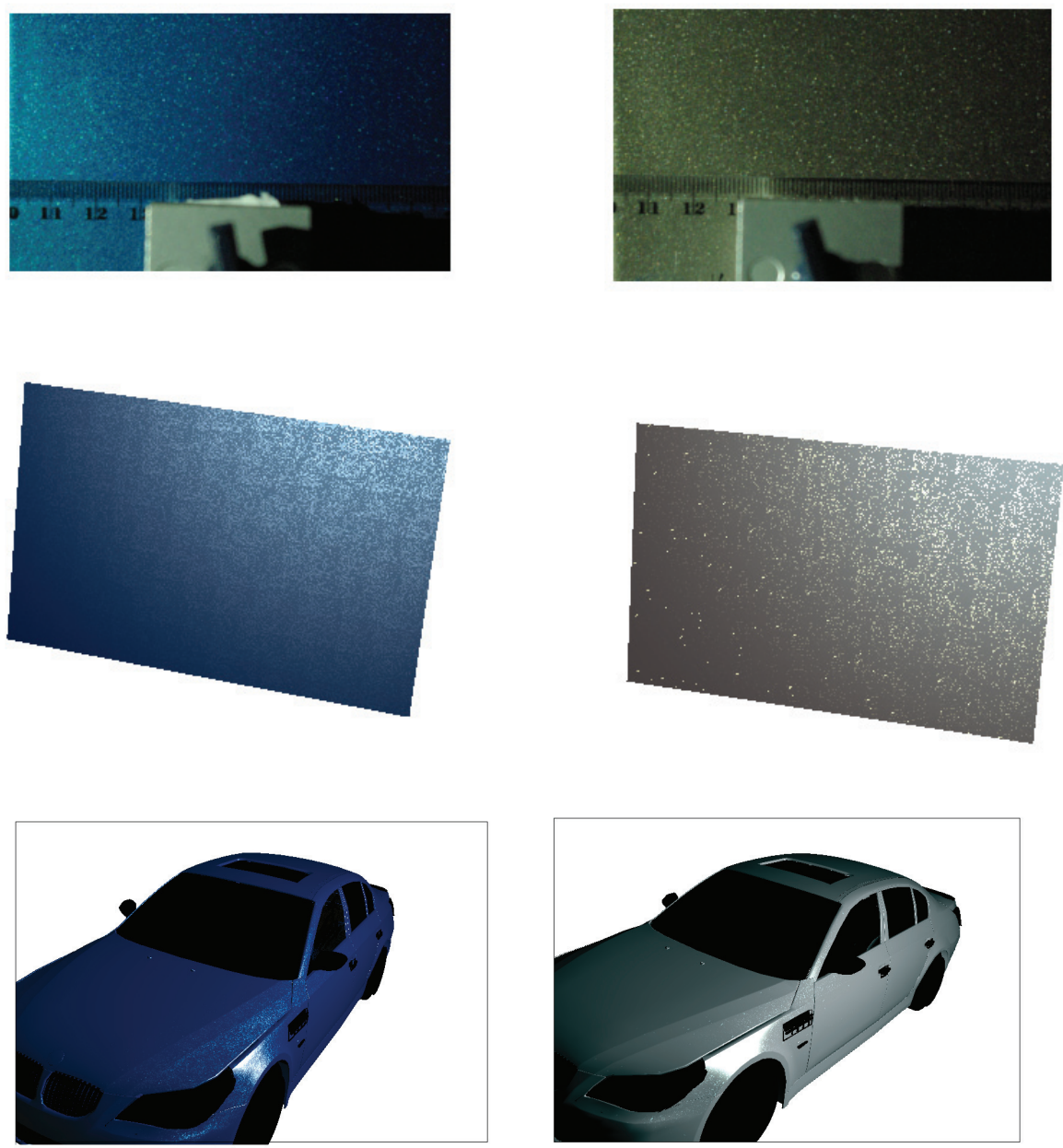

Fig. 7. Left column: Neysha. Right column: Cendré. First row: sample photograph. Middle row: rendered planar sample. Bottom row: renderd car model.

\section{CONCLUSION}

We set up measuring device to BRDF acquisition of real materials. The device consists of common laboratory equipments. We have experimentally studied simple materials and compared the obtained results to simulations based on the analytical BRDF. We simulated the rendering results to validate acquired reflectance model obtained by fitting process from measurements of real samples. The sample surface is represneted by Cook-Torrance model for particular wavelength with estimated parameters. We found out that it is possible to get plausible results despite the sparse angular resolution of measurement using suitable model and fitting method.

To achieve effects such as sparkling of metallic paints we made multiple photographs of 


\section{R. ĎURIKOVIČ, A. MIHÁLIK}

the surface under the variety of light incidence. We created the sparkle texture where directional light effects were incorporated. Finally, we rendered surface color using GPU. This process consisted of spectrum transformation to RGB color and adding sparkle intensity according to the sparkle texture.

\section{ACKNOWLEDGMENT}

We would like to thank to Dušan Chorvát from ILC for his help with laboratory measurements.

\section{REFERENCES}

Achutha, S. 2006. Brdf acquisition with basis illumination. M.S. thesis, Department of Computer Science, University of British Columbia, Vancouver, Canada.

Ashikhmin, M. And Shirley, P. 2000. An anisotropic phong BRDF model. J. Graph. Tools 5, 2 (Feb.), $25-32$.

Bagher, Mahdi, M., Soler, C., ANd Holzschuch, N. 2012. Accurate fitting of measured reflectances using a Shifted Gamma micro-facet distribution. Computer Graphics Forum 31, 4 (June).

BJORCK, A. 1996. Numerical Methods for Least Squares Problems, 1 ed. SIAM: Society for Industrial and Applied Mathematics.

BochKAnOv, S. AND Bystritsky, V. 2013. Least squares fitting. ALGLIB (www.alglib.net).

CoOK, R. L. AND TORRANCE, K. E. 1982. A reflectance model for computer graphics. ACM Trans. Graph. 1, 1, 7-24.

ĎURIKOVIČ, R. 2002. Explicit method of sparkling effect simulation. Journal of Three Dimensional Images 16, 4, 96-100.

ISO2813. 1994. Paints and varnishes -Determination of specular gloss of non-metallic paint films at 20 degrees, 60 degrees and 85 degrees. Internation Organization for Standardization.

Kim, D. B., Kim, K. Y., PARK, K. S., SeO, M. K., AND LeE, K. H. 2008. A fast and accurate image-based measuring system for isotropic reflection materials. Z.-H. Gu and L. M. Hanssen, Eds. Reflection, Scattering, and Diffraction from Surfaces 7065, 1, 70650I.

Matusik, W., Pfister, H., Brand, M., And McMillan, L. 2003. A data-driven reflectance model. In SIGGRAPH '03: ACM SIGGRAPH 2003 Papers. ACM, New York, NY, USA, 759-769.

MiHÁLIK, A. AND ĎURIKOVIČ, R. 2011. Virtual gonio-spectrophotometer for validation of BRDF designs. Central European Journal of Physics 9, 1334-1343.

NGan, A., Durand, F., AND MAtusik, W. 2005. Experimental analysis of BRDF models. In Proceedings of the Sixteenth Eurographics conference on Rendering Techniques. EGSR'05. Eurographics Association, Airela-Ville, Switzerland, Switzerland, 117-126.

Rump, M., Müller, G., Sarlette, R., Koch, D., ANd Klein, R. 2008. Photo-realistic rendering of metallic car paint from image-based measurements. Computer Graphics Forum 27, 2 (Apr.).

RushmeIER, H. 2001. Global illumination. In Wiley Encyclopedia of Electrical and Electronics Engineering. John Wiley \& Sons, Inc.

Sergey Ershov, Andrei Khodulev, K. K. 1999. Simulation of sparkles of metallic paints. In Proceeding of Graphicon. 121-128.

Tonsho, K., Akao, Y., Tsumura, N., And MiYake, Y. 2001. Development of goniophotometric imaging system for recording reflectance spectra of 3D objects. In Society of Photo-Optical Instrumentation Engineers (SPIE) Conference Series, R. Eschbach and G. G. Marcu, Eds. Society of Photo-Optical Instrumentation Engineers (SPIE) Conference Series, vol. 4663. 370-378.

TORRAnCE, K. E. AND Sparrow, E. M. 1967. Theory for off-specular reflection from roughened surfaces. JOSA 57, 9, 1105-1114.

Ďurikovič, R. AND MARTENS, W. L. 2003. Simulation of sparkling and depth effect in paints. In SCCG '03: Proceedings of the 19th spring conference on Computer graphics. ACM, New York, NY, USA, 193-198.

WARD, G. J. 1992. Measuring and modeling anisotropic reflection. SIGGRAPH Comput. Graph. 26, 2, $265-272$. 
Authors' addresses:

Andrej Mihálik

Mathematics, Physics and Informatics,

Comenius University,

84248 Bratislava, Slovak Republic

http://www.fmph.uniba.sk

email: mihalik@sccg.sk

Roman Ďurikovič

Faculty of Mathematics, Physics and Informatics,

Comenius University,

84248 Bratislava, Slovak Republic

http://www.fmph. uniba.sk

email: roman.durikovic@fmph.uniba.sk

Received October 2013 